\title{
SOME CHARACTERS OF THE SYMMETRIC GROUP
}

\author{
R. E. INGRAM, S.J.
}

Introduction. Frobenius [1] ${ }^{1}$ derived expressions for the characters of a few very simple classes of $S_{m}$, the symmetric group on $m$ things. Here we give formulas for some more complicated classes. The method is quite general. A recurrence formula, due to Murnaghan, is used. The need for the formulas arose from general considerations of nuclear binding forces. ${ }^{2}$ Some applications will be given in another paper.

1. The character $\chi(p)_{\lambda}$, of the class $\alpha_{1}=m-p, \alpha_{p}=1[1,2]$. If $\{\lambda\}$, in the usual notation, is a partition of $m$, the same partition may also be represented [6] by two sets of numbers $\{b, a\}$ and the following relation holds,

$$
\sum_{j=1}^{k}\left(\lambda_{j}-j\right)^{n}=\sum_{j=1}^{s} b_{i}^{n}+(-1)^{n+1} \sum_{j=1}^{s}\left(a_{j}+1\right)^{n}+(-1)^{n} \sum_{j=1}^{k} j^{n}
$$

If $\chi_{\lambda}$ is the character of a class $K$ of $S_{m}$,

$$
\left\{\lambda_{j}^{\prime}\right\}=\left\{\lambda_{1}, \lambda_{2}, \cdots, \lambda_{j}-p, \cdots, \lambda_{k}\right\}
$$

a partition of $m-p$, and $\chi_{\lambda_{i}^{\prime}}$ the character of the class $K^{\prime}$ of $S_{m-p}$, where $K^{\prime}$ has the same cyclic structure as $K$, but with one less $p$-cycle, then

$$
\chi_{\lambda}=\sum_{j=1}^{k}{\chi \lambda_{j}^{\prime}} \quad \text { (Murnaghan Recurrence Formula [6]). }
$$

Denoting by $\chi(p)_{\lambda}$ the character of the class having one $p$-cycle (all others unary), we have

$$
\chi(p)_{\lambda}=\sum_{1}^{k} \chi \lambda_{j}^{\prime}=\sum_{1}^{k} D_{\lambda_{j}^{\prime}}
$$

where $D$ is the dimension of the representation.

$$
\begin{aligned}
& D_{\lambda_{j}^{\prime}}=\frac{(m-p) ! \Delta\left(l_{j}^{\prime}\right)}{l_{1} ! l_{2} ! \cdots\left(l_{j}-p\right) ! \cdots l_{k} !}, \\
& \quad\left(\Delta(l)=\prod_{p<q}\left(l_{p}-l_{q}\right), l_{p}=\lambda_{p}+k-p\right)
\end{aligned}
$$

Received by the editors April 24, 1949.

1 Numbers in brackets refer to bibliography at end of this paper.

2 The original problem to find $x(2,2) \lambda$ is due to Dr. J. A. Wheeler, Princeton [7]. 


$$
\begin{aligned}
& =\frac{(m-p) !}{m !} \frac{l_{j} !}{\left(l_{j}-p\right) !} \frac{f\left(l_{j}-p\right)}{-p f^{\prime}\left(l_{j}\right)} \quad\left(\text { where } f(x)=\prod_{i=1}^{k}\left(x-b_{i}\right)\right) \\
& =\frac{(m-p) !}{m !} D_{\lambda}\left[x(x-1) \cdots(x-p+1) \frac{f(x-p)}{f(x)}\right]_{x=l_{j}} .
\end{aligned}
$$

This expression for $D_{\lambda_{j}^{\prime}}$ may be written as $\sum_{1}^{\mathbf{k}} A_{j} /\left(x-\lambda_{j}\right)$ plus a polynomial in $x$, where $A_{j}=l_{j}\left(l_{j}-1\right) \cdots\left(l_{j}-p+1\right) f\left(l_{j}-p\right) / f^{\prime}\left(l_{j}\right)$. If we expand $\sum_{1}^{k} A_{j} /\left(x-l_{j}\right)$ in descending powers of $x$, it is evident that $\sum_{1}^{k} A_{j}$ is the coefficient of $1 / x$ in the expansion of

$$
x(x-1) \cdots(x-p+1) \frac{f(x-p)}{f(x)}
$$

in descending powers of $x$.

Likewise

$$
\sum_{1}^{k} l_{j} A_{j} \quad \text { is the coefficient of } 1 / x^{2}
$$

and

$$
\sum_{1}^{k} l_{j}^{\alpha} A_{i} \quad \text { is the coefficient of } 1 / x^{\alpha+1}
$$

Thus

$$
\sum_{1}^{k} l_{j}^{\alpha} D_{\lambda_{j}^{\prime}}=-\frac{m-p !}{m !} D_{\lambda} \frac{1}{p} \sum_{1}^{k} l_{j}^{\alpha} A_{j}
$$

and taking $\alpha=0$, we have

$$
\begin{aligned}
\chi(p)_{\lambda} & =\sum_{1}^{k} D_{\lambda_{j}^{\prime}}=\frac{m-p !}{m !} D_{\lambda}\left(\frac{-1}{p}\right) \sum_{1}^{k} A_{j}, \\
\frac{m !}{m-p !} \frac{\chi(p)_{\lambda}}{D_{\lambda}} & =\left[x(x-1) \cdots(x-p+1) \frac{f(x-p)}{-p f(x)}\right]_{1 / x} .
\end{aligned}
$$

It should be noted that if $\left\{\lambda_{j}^{\prime}\right\}$ ends in a negative number when arranged in non-ascending order [6], then $\lambda_{j}-p+k-j<0$, as $\lambda_{j}-p$ may be increased by $k-j$ by moving down to $k$ th position from $j$ th position. Therefore

$$
l_{j}-p<0,
$$

3 [ ] $]_{1 / x}$ signifies the coefficient of $1 / x$ in the expansion of the function within the brackets. 


$$
l_{i}-p+1 \leqq 0 .
$$

Therefore

$$
A_{j}=0 .
$$

If $\left\{\lambda_{j}^{\prime}\right\}$ cannot be rearranged to form a non-increasing sequence

$$
\begin{aligned}
\lambda_{i}-p & =\lambda_{j+\infty}-S, \\
\lambda_{i}+j-K-p & =\lambda_{j+e}+j-K-S, \\
l_{i}-p & =l_{j+\bullet} .
\end{aligned}
$$

some $S$,

Therefore

$$
f\left(l_{j}-p\right)=0, A_{j}=0 .
$$

In neither case is any contribution made to $\sum_{1}^{k} A_{j}$.

Let $x=y+k^{\prime}$, then

$$
\begin{aligned}
{[x(x-1) \cdots(x-} & \left.p+1) \frac{f(x-p)}{f(x)}\right]_{1 / x} \\
& =\left[y(y-1) \cdots(y-p+1) \frac{F(y-p)}{F(y)}\right]_{1 / y}
\end{aligned}
$$

where

$$
F(y)=\prod_{j=1}^{\dot{1}}\left[\frac{\left(y-b_{i}\right)}{\left(y+a_{j}+1\right)}\right], \quad a, b \text { as in (1.1), }
$$

and

$$
\begin{aligned}
\frac{F(y-p)}{-p F(y)}= & -1 / p+\frac{m_{2}}{y}+\frac{1}{y^{2}}\left(c_{3}+p m\right) \\
& +\frac{1}{y^{4}}\left(c_{4}+\frac{3}{2} p c_{3}+p^{2} m-\frac{p}{2} m^{2}\right) \\
& +\frac{1}{y^{6}}\left(c_{6}+2 p c_{4}-p m c_{3}+2 p^{2} c_{3}-p^{2} m^{2}+p^{2} m\right) \\
& +\frac{1}{y^{6}}\left(c_{6}-\frac{p}{2} c_{3}^{2}-p m c_{4}+\frac{5}{2} p c_{5}-\frac{5}{2} p^{2} m c_{3}\right. \\
& \left.+\frac{10}{3} p^{2} c_{4}+\frac{p^{2} m^{2}}{6}-\frac{3}{2} m^{2} p^{2}+\frac{5}{2} p^{2} c_{3}+m p^{4}\right) \\
& +\cdots
\end{aligned}
$$


with

$$
\begin{aligned}
m & =\sum_{1}^{\dot{1}}\left\{b_{j}+\left(a_{j}+1\right)\right\}, \\
c_{2 r+1} & =\sum_{1}^{\dot{1}}\left\{b_{i}^{2 r}-\left(a_{i}+1\right)^{2 r}\right\}, \\
c_{2 r} & =\sum_{1}^{\dot{1}}\left\{b_{i}^{2 r+1}+\left(a_{i}+1\right)^{2 r}\right\} .
\end{aligned}
$$

Using (1.7) and (1.8) in (1.6), we get the expressions (5.1), (5.2), (5.3), (5.4), (5.5) on pp. 367-368.

2. The character $\chi(p, 2)_{\lambda}$ of the class $\alpha_{1}=m-p-2, \alpha_{2}=1, \alpha_{p}=1$. Let $\chi(p, 2)_{\lambda}$ be the character of the class $\alpha_{2}=1$ binary cycle and $\alpha_{p}=1$ cycle on $p$ letters.

By the recurrence formula, (1.2),

$$
x(p, 2)_{\lambda}=\sum_{j=1}^{k} x(2)_{\lambda_{j}^{\prime}}
$$

where $\lambda_{j}^{\prime}$, as a subscript, indicates that $\chi(2)$ is the character of the representation $\left\{\lambda_{j}^{\prime}\right\}=\left\{\lambda_{1}, \lambda_{2}, \cdots, \lambda_{j}-p_{1}, \cdots, \lambda_{k}\right\}$ of the symmetric group on $m-p$ letters, $S_{m-p}$, corresponding to the class $\alpha_{1}$ $=m-p-2$ unary cycles, $\alpha_{2}=1$ binary cycle. By (5.2),

$$
\chi(2)_{\lambda_{j}^{\prime}}=\left(M_{2}\right)_{j}^{\prime} D_{\lambda_{j}}(m-p-2) ! /(m-p) \text { ! }
$$

where $\left(M_{2}\right)_{j}^{\prime}$ is $M_{2}$ with $\lambda_{j}-p$ instead of $\lambda_{j}$ (that is, the correct value of $M_{2}$ for $\left.\left\{\lambda_{j}^{\prime}\right\}\right)$.

$D_{\lambda_{j}^{\prime}}$ is the dimension of $\left\{\lambda_{j}^{\prime}\right\}$, and $m-p$ replaces $m$ as $\left\{\lambda_{j}^{\prime}\right\}$ is a representation of $S_{m-p}$.

$$
M_{2}=\sum_{j=1}^{h}\left(\lambda_{i}-j\right)\left(\lambda_{i}-j+1\right)-\sum_{1}^{h} j^{2}+\sum_{1}^{k} j
$$

is a function of $\lambda_{1}, \lambda_{2}, \cdots, \lambda_{k}$.

$\left(M_{2}\right)_{j}^{\prime}$ is the value for $\lambda_{1}, \lambda_{2}, \cdots,\left(\lambda_{j}-p\right), \cdots, \lambda_{k}$,

$$
\left(M_{2}\right)_{i}^{\prime}=M_{2}-2 p\left(\lambda_{i}-j\right)+p(p-1) \text {. }
$$

Therefore

$$
\chi(2)_{\lambda_{j^{\prime}}}=\frac{M_{2}+p(p-1)-2 p\left(\lambda_{j}-j\right)}{(m-p)(m-p-1)} D_{\lambda_{j^{\prime}}}
$$

Then, (2.1), 


$$
\begin{aligned}
\chi(p, 2)_{\lambda}= & \sum_{1}^{k} \frac{M_{2}+p(p-1)-2 p\left(\lambda_{j}-j\right)}{(m-p)(m-p-1)} D_{\lambda_{j}^{\prime}} \\
= & \frac{M_{2}+p(p-1)}{(m-p)(m-p-1)} \sum_{1}^{k} D_{\lambda_{i}^{\prime}} \\
& -\frac{2 p}{(m-p)(m-p-1)} \sum_{1}^{k}\left(\lambda_{i}-j\right) D_{\lambda_{j}^{\prime}}
\end{aligned}
$$

(since $M_{2}, p, m$ are the same in all terms) $\chi(p)_{\lambda}=\sum_{1}^{k} D_{\lambda_{j}}$ has already been found.

$$
\begin{aligned}
\sum_{1}^{k}\left(\lambda_{i}-j\right) D_{\lambda_{j}^{\prime}} & =\sum_{1}^{k}\left(l_{j}-k\right) D_{\lambda_{j}^{\prime}} \\
& =\sum_{1}^{k} l_{j} D_{\lambda_{j}^{\prime}}-k \sum_{1}^{k} D_{\lambda_{j}^{\prime}}, \\
\sum_{1}^{k} l_{j} D_{\lambda_{j}^{\prime}} & =\frac{m-p !}{m !} D_{\lambda}\left(-\frac{1}{p} \sum_{1}^{k} l_{j} A_{i}\right)
\end{aligned}
$$

where

$$
\sum_{1}^{k} l_{j} A_{j}=\left[x(x-1) \cdots(x-p+1) \frac{f(x-p)}{f(x)}\right]_{1 / x^{2}}
$$

(that is, the coefficient of $1 / x^{2}$ in the expansion of $x(x-1) \cdots$ $(x-p+1) f(x-p) / f(x))$. Equivalently

$$
\sum_{1}^{k} l_{j} A_{i}=\left[x^{2}(x-1) \cdots(x-p+1) \frac{f(x-p)}{f(x)}\right]_{1 / x} .
$$

Putting, as in (1.7), $x=y+k$, we have

$$
\begin{aligned}
{\left[x^{2}(x-1) \cdots\right.} & \left.(x-p+1) \frac{f(x-p)}{f(x)}\right]_{1 / x} \\
& =\left[(y+k) y(y-1) \cdots(y-p+1) \frac{F(y-p)}{F(y)}\right]_{1 / y}, \\
\sum_{1}^{k} D_{\lambda_{j^{\prime}}} & =\left[y(y-1) \cdots(y-p+1) \frac{F(y-p)}{-p F(y)}\right]_{1 / y} \frac{m-p !}{m !} D_{\lambda .}
\end{aligned}
$$

Therefore

(2.3) $\sum_{1}^{k}\left(\lambda_{i}-j\right) D_{\lambda_{j}^{\prime}}=\sum_{1}^{k} l_{j} D_{\lambda_{j}^{\prime}}-k \sum_{1}^{k} D_{\lambda_{j}^{\prime}}$ 


$$
\begin{aligned}
= & \left\{\left[(y+k) y \cdots(y-p+1) \frac{F(y-p)}{F(y)}\right]_{1 / y}\right. \\
& \left.-k\left[y \cdots(y-p+1) \frac{F(y-p)}{F(y)}\right]_{1 / y}\right\} \frac{m-p !}{m !} D_{\lambda} \\
= & {\left[y^{2}(y-1) \cdots(y-p+1) \frac{F(y-p)}{-p F(y)}\right]_{1 / y} \frac{m-p !}{m !} D_{\lambda} . }
\end{aligned}
$$

From the general expression (2.2) the formulas for $\chi(2,2), \chi(3,2)$, $\chi(4,2)$ are obtained, for example formula for $\chi(2,2)$ (that is, $p=2)$ :

By (2.2),

$$
\begin{aligned}
& \chi(2,2)_{\lambda}=\frac{\left(M_{2}+2\right)}{(m-2)(m-3)} \sum_{1}^{k} D_{\lambda_{j}^{\prime}}-\frac{4}{(m-2)(m-3)} \sum_{1}^{k}\left(\lambda_{i}-j\right) D_{\lambda_{j}^{\prime}}, \\
& \sum_{1}^{k} D_{\lambda_{j}^{\prime}}=\chi(2)_{\lambda}=\frac{M_{2} D_{\lambda}}{m(m-1)}, \\
& \sum_{1}^{k}\left(\lambda_{j}-j\right) D_{\lambda_{j}^{\prime}}=\frac{D_{\lambda}}{m(m-1)}\left[y^{2}(y-1) \frac{F(y-2)}{-2 F(y)}\right]_{1 / y} .
\end{aligned}
$$

Using (1.3) with $p=2$, multiplying by $y^{2}(y-1)$, and taking the coefficient of $1 / y$, we have

$$
\sum_{1}^{k}\left(\lambda_{i}-j\right) D_{\lambda_{j}^{\prime}}=\frac{D_{\lambda}\left(c_{4}+2 c_{3}+2 m-m^{2}\right)}{m(m-1)(m-2)(m-3)}
$$

hence

$$
\begin{aligned}
\chi(2,2)_{\lambda} & =\frac{\left(M_{2}+2\right) M_{2}-4\left(c_{4}+3 c_{3}+4 m-m^{2}\right)+4\left(c_{3}+2 m\right)}{m(m-1)(m-2)(m-3)} D_{\lambda} \\
& =\frac{M_{2}^{2}-2 M_{3}+4 m(m-1)}{m(m-1)(m-2)(m-3)} D_{\lambda}
\end{aligned}
$$

where $M_{2}, M_{3}$ are as defined previously.

3. The character $\chi(p, 3)$. Let $\chi(p, 3)$ be the character of the class $\alpha_{1}=m-p-3$ unary cycles, $\alpha_{3}=1$ ternary cycle (cycle on 3 letters) and $\alpha_{p}=1$ cycle on $p$ letters.

By the recurrence formula (1.2),

$$
\chi(p, 3)=\sum_{1}^{k} \chi(3)_{\lambda_{j}^{\prime}}
$$


where $\lambda_{j}^{\prime}$, as a subscript, indicates that $\chi(3)$ is the character of a representation $\left\{\lambda_{j}^{\prime}\right\}=\left\{\lambda_{1}, \lambda_{2}, \cdots,\left(\lambda_{j}-p\right), \cdots, \lambda_{k}\right\}$ of $S_{m-p}$ corresponding to the class obtained from the original class by dropping the cycle on $p$-letters; it is, then, the character of a class with one ternary cycle and all other cycles unary.

$\chi(3)_{\lambda_{j}}$ is known, it is given by $\$ 1$, cf. (5.2)

$$
\chi(3)_{\lambda_{j^{\prime}}}=\left[1 / 2\left(M_{3}\right)_{i}^{\prime}-3 / 2(m-p)(m-p-1)\right] \frac{m-p-3 !}{m-p !} D_{\lambda_{j}^{\prime}}
$$

where $\left(M_{3}\right)_{j}^{\prime}$ is the same as $M_{3}$ but has $\lambda_{j}-p$ in place of $\lambda_{j}, m$ is replaced by $m-p$ as $\left\{\lambda_{j}^{\prime}\right\}$ is a representation of $S_{m-p}$, and $D_{\lambda_{j}^{\prime}}$ is the dimension of $\left\{\lambda_{j}^{\prime}\right\}$.

$$
M_{8}=\sum_{1}^{k}\left[2\left(\lambda_{i}-j\right)^{3}+3\left(\lambda_{i}-j\right)^{2}+\left(\lambda_{i}-j\right)+2 j^{3}-3 j^{2}+j\right]
$$

is a function of $\lambda_{1}, \lambda_{2}, \cdots, \lambda_{k}$. $\left(M_{3}\right)_{j}^{\prime}$ is found by substituting $\lambda_{j}-p$ for $\lambda_{j}$,

$$
\begin{array}{r}
\left(M_{3}\right)_{j}^{\prime}=M_{3}-6 p\left(\lambda_{j}-j\right)^{2}+6 p(p-1)\left(\lambda_{j}-j\right)-p^{2}(2 p-3) . \\
x(3)_{\lambda_{j}^{\prime}}=\left[1 / 2 M_{3}-3 p\left(\lambda_{j}-j\right)^{2}+3 p(p-1)\left(\lambda_{i}-j\right)-p^{2} / 2(2 p-3)\right. \\
-3 / 2(m-p)(m-p-1)] D_{\lambda_{j}^{\prime}} \frac{(m-p-3) !}{m-p !}
\end{array}
$$

and by (3.1)

$$
\begin{aligned}
x(p, 3)_{\lambda}=\sum_{1}^{k}\left[\frac{1}{2} M_{3}-3 p\left(\lambda_{j}-j\right)^{2}+3 p(p-1)\left(\lambda_{j}-j\right)\right. \\
\left.-\frac{p^{2}}{2}(2 p-3)-\frac{3}{2}(m-p)(m-p-1)\right] D_{\lambda_{j}^{\prime}} \frac{(m-p-3) !}{m-p !} \\
=\left[\frac{1}{2} M_{3}-p^{2} / 2(2 p-3)\right. \\
\left.\quad-\frac{3}{2}(m-p)(m-p-1)\right] \frac{(m-p-3) !}{m-p !} \sum_{1}^{k} D_{\lambda_{j}^{\prime}} \\
\quad-3 p \sum_{1}^{k}\left[\left(\lambda_{i}-j\right)^{2}-(p-1)\left(\lambda_{j}-1\right)\right] \frac{m-p-3 !}{m-p !} D_{\lambda_{j}^{\prime}} .
\end{aligned}
$$

$\sum_{j=1}^{k} D_{\lambda_{j}^{\prime}}=\chi(p)$ and is known by the results of $\$ 1$. 


$$
\begin{aligned}
\sum_{1}^{k}\left[\left(\lambda_{j}-j\right)^{2}-(p-1)\left(\lambda_{j}-j\right)\right] & D_{\lambda_{j}^{\prime}} \\
& =\sum_{1}^{k}\left[\left(l_{j}-k\right)^{2}-(p-1)\left(l_{j}-k\right)\right] D_{\lambda_{j}^{\prime}}
\end{aligned}
$$

where $\lambda_{j}-j=l_{j}-k$ as previously in $\$ 1$.

$$
\begin{aligned}
\sum_{1}^{k}\left(l_{j}-k\right) D_{\lambda_{j}^{\prime}} & \\
= & {\left[y^{2}(y-1) \cdots(y-h+1) \frac{F(y-h)}{-p F(y)}\right]_{1 / y} \frac{m-p !}{m !} D_{\lambda,} } \\
& \sum_{1}^{k} l_{j}^{2} D_{\lambda_{j}^{\prime}}=\frac{m-p !}{m !} D_{\lambda}\left(-1 / p \sum_{1}^{k} l_{j}^{2} A_{j}\right),
\end{aligned}
$$

as in $\$ 1$, where $A_{j}=l_{j}\left(l_{j}-1\right) \cdots\left(l_{j}-p+1\right) f\left(l_{j}-p\right) / f^{\prime}\left(l_{j}\right)$ and $f(x)$ $=\coprod_{1}^{k}\left(x-l_{j}\right)$ and

$$
\begin{aligned}
\sum_{1}^{k} l_{j}^{2} A_{j}= & {\left[x(x-1) \cdots(x-p+1) \frac{f(x-p)}{f(x)}\right]_{1 / x^{2}} } \\
= & {\left[x^{3}(x-1) \cdots(x-p+1) \frac{f(x-p)}{f(x)}\right]_{1 / x} } \\
= & {\left.\left[(y+k)^{2} y(y-1) \cdots(y-p+1) \frac{F(y-p)}{F(y)}\right]_{1 / y}\right]^{\prime} } \\
\sum_{1}^{k}\left(l_{j}-k\right)^{2} A_{i}= & {\left[\left\{(y+k)^{2}-2 k(y+k)+k^{2}\right\} y(y-1)\right.} \\
= & {\left[y^{2}(y-1) \cdots(y-p+1) \frac{F(y-p)}{F(y)}\right]_{1 / y}, } \\
\sum_{1}^{k}\left(\lambda_{i}-j\right)^{2} D_{\lambda_{j}^{\prime}}= & {\left[y^{3}(y-1) \cdots(y-p+1) \frac{F(y-p)}{-p F(y)}\right]_{1 / y} } \\
& \cdot \frac{m-p !}{m !} D_{\lambda \cdot} .
\end{aligned}
$$

From these expressions, $\chi(p, 3)_{\lambda}$ is obtained. (If $p=2$ the character $\chi(2,3)$ is obtained, it is of course the same expression as in \$2.) 
Taking $p=3$, we have

$$
\begin{gathered}
\chi(3,3)_{\lambda}=\left[1 / 2 M_{3}-27 / 2-3 / 2(m-3)(m-4)\right] \frac{m-6 !}{m-3 !} \sum_{1}^{k} D_{\lambda_{j}^{\prime}} \\
-9 \sum_{1}^{k}\left[\left(\lambda_{j}-j\right)^{2}-2\left(\lambda_{j}-j\right)\right] D_{\lambda_{j}^{\prime}} \frac{m-6 !}{m-3 !} \\
\sum_{1}^{k} D_{\lambda_{j}^{\prime}}=\chi(3)=\frac{\left[1 / 2 M_{3}-3 / 2 m(m-1)\right] D_{\lambda}}{m(m-1)(m-2)} \\
\sum_{1}^{k}\left[\left(\lambda_{j}-j\right)^{2}-2\left(\lambda_{j}-j\right)\right] D_{\lambda_{j}^{\prime}} \\
=\frac{D_{\lambda}}{m(m-1)(m-2)}\left[y^{2}(y-1)(y-2)^{2} \frac{F(y-3)}{-3 F(y)}\right]_{1 / y}
\end{gathered}
$$

Using the expansion of $F(y-3) /-3 F(y)$ given by (1.8) with $p=3$, multiplied by $y^{2}(y-1)(y-2)^{2}$, and collecting the coefficient of $1 / y$, finally we obtain the expression for $\chi(3,3)$,

$$
\begin{aligned}
\chi(3,3)=\left[1 / 4 M_{3}^{2}-18 M_{5}\right. & +54 M_{3}^{2}-6\left(m^{2}-13 m-39\right) M_{3} \\
& \left.+9 m(m-1)\left(m^{2}-13 m+34\right)\right] D_{\lambda} \frac{m-6 !}{m !},
\end{aligned}
$$

with $M_{2}, M_{3}, M_{5}$ as defined previously.

4. The character $\chi(2,2,2)_{\lambda}$. It is evident that, by means of the recurrence formula, formulas can be found for characters of classes consisting of three non-unary cycles. The expressions involved, however, become too complicated for easy computation. To illustrate the method it is sufficient to take the simplest case, the class consisting of three binary cycles and $m-6$ unary cycles.

By the recurrence formula (1.2),

$$
\chi(2,2,2)_{\lambda}=\sum_{j=1}^{k} \chi(22)_{\lambda_{j^{\prime}}}
$$

where $\chi(2,2,2)_{\lambda}$ is the required character of the representation $\{\lambda\}$ of $S_{m}, \chi(2,2)_{\lambda_{j}^{\prime}}$ is the character of the class, consisting of 2 binary cycles and $m-6$ unary cycles, of $S_{m-2}$, and $\left\{\lambda_{j}^{\prime}\right\}$ is the representation $\left\{\lambda_{1}, \lambda_{2}, \cdots, \lambda_{j}-2, \cdots, \lambda_{k}\right\}$ of $S_{m-2} \chi(2,2)_{\lambda_{j}^{\prime}}$ is known, it is given by (2.3) or (5.5). 
1950]

$$
\chi(2,2)_{\lambda_{j}^{\prime}}=\sum_{j=1}^{k} \frac{\left[\left(M_{2}\right)_{j}^{\prime}\right]^{2}-2\left(M_{3}\right) i+4(m-2)(m-3)}{(m-2)(m-3)(m-4)(m-5)} .
$$

$\left(M_{2}\right)_{j}^{\prime}$ is $M_{2}$ written for $\lambda_{j}^{\prime}$, that is, $\lambda_{j}-2$ is written for $\lambda_{j}$, as $M_{2}$ $=\sum_{1}^{k} \lambda_{j}\left(\lambda_{j}-2 j+1\right)$.

$$
\begin{aligned}
{\left[\left(M_{2}\right)_{j}^{\prime}\right]^{2}=} & M_{2}^{2}-8\left(\lambda_{j}-j\right) M_{2}+4 M_{2}+16\left(\lambda_{j}-j\right)^{2} \\
& -16\left(\lambda_{j}-j\right)+4 \\
\left(M_{3}\right)_{j}^{\prime}= & M_{3}-12\left(\lambda_{j}-j\right)^{2}+12\left(\lambda_{j}-j\right)-6
\end{aligned}
$$

$$
\begin{aligned}
\chi(2,2,2)= & \sum_{1}^{k}\left[M_{2}^{2}+4 M_{2}-2 M_{8}+4 m^{2}-20 m+40\right] D_{\lambda_{j}^{\prime}} \frac{m-6 !}{m-2 !} \\
& +\sum_{1}^{k}\left[40\left(\lambda_{j}-j\right)^{2}-8\left(M_{2}+5\right)\left(\lambda_{j}-j\right) D_{\lambda_{j}^{\prime}}\right] \frac{m-6 !}{m-2 !}
\end{aligned}
$$

The first of these two sums is $\left[M_{2}^{2}+4 M_{2}-2 M_{3}+4 m^{2}-20 m\right.$ $+40] M_{2} D_{\lambda}(m-6) ! / m !$. The second is obtained by the same reasoning as in the previous sections,

$$
\begin{aligned}
\sum_{1}^{k}\left[4 0 \left(\lambda_{j}\right.\right. & \left.-j)^{2}-8\left(M_{2}+5\right)\left(\lambda_{j}-j\right)\right] D_{\lambda_{j}^{\prime}} \\
& =\left[\left(40 y^{2}-8\left(M_{2}+5\right) y\right) y(y-1) \frac{F(y-2)}{-2 F(y)}\right]_{1 / y} D_{\lambda} \cdot \frac{m-6 !}{m !} \\
& =\left[8 y\left(y-M_{2}-5\right) y(y-1) \frac{F(y-2)}{-2 F(y)}\right]_{1 / y} D_{\lambda} \cdot \frac{m-6 !}{m !} .
\end{aligned}
$$

The expansion of $F(y-2) /-2 F(y)$ is found from (1.8), and taking the coefficient of $1 / y$ when this series is multiplied by $8 y^{2}(y-1)$ $\cdot\left(y-M_{2}-5\right)$, we get

$\chi(2,2,2)_{\lambda}=\left[M_{2}^{3}-6 M_{2} M_{3}+40 M_{4}+12\left(m^{2}-9 m+10\right) M_{2}\right] D_{\lambda} \frac{m-6 !}{m !}$.

5. Results.

$$
\frac{\chi(2)_{\lambda}}{D_{\lambda}} \frac{m !}{m-2 !}=M_{2}
$$

$$
\begin{aligned}
& \frac{\chi(3)_{\lambda}}{D_{\lambda}} \frac{m !}{m-3 !}=\frac{M_{8}}{2}-3 / 2 m(m-1) \\
& \frac{\chi(4)_{\lambda}}{D_{\lambda}} \frac{m !}{m-4 !}=M_{4}-2(2 m-3) M_{2}
\end{aligned}
$$

(Frobenius),

(Frobenius), 


$$
\begin{aligned}
& \text { (5.4) } \frac{\chi(5)_{\lambda}}{D_{\lambda}} \frac{m !}{m-5 !}=\frac{M_{5}}{2}+\frac{49-15 m}{6} M_{3}-5 / 2 M_{2}^{2} \\
& +5 / 6 m(m-1)(5 m-19), \\
& \text { (5.5) } \frac{\chi(2,2)_{\lambda}}{D_{\lambda}} \frac{m !}{m-4 !}=M_{2}^{2}-2 M_{3}+4 m(m-1) \text {, } \\
& \frac{\chi(3,2)_{\lambda}}{D_{\lambda}} \frac{m !}{m-5 !}=\frac{1}{2}\left[M_{2} M_{3}-6 M_{4}-3 / 2\left(m^{2}-13 m+16\right) M_{2}\right] \text {, } \\
& \frac{\chi(4,2)_{\lambda}}{D_{\lambda}} \frac{m !}{m-6 !}=M_{2} M_{4}-4 M_{6}-2(2 m-11) M_{2}^{2} \\
& +4 / 3(12 m-34) M_{3} \\
& -32 / 3 m(m-1)(2 m-7) \text {, } \\
& \frac{\chi(3,3)_{\lambda}}{D_{\lambda}} \frac{m !}{m-6 !}=\frac{1}{4}\left[M_{3}^{2}-18 M_{6}+54 M_{2}^{2}\right. \\
& -6\left(m^{2}-13 m-39\right) M_{3} \\
& \left.+9 m(m-1)\left(m^{2}-13 m+34\right)\right] \text {, } \\
& \frac{\chi(2,2,2)}{D_{\lambda}} \frac{m !}{m-6 !}=M_{2}^{3}-6 M_{2} M_{3}+40 M_{4} \\
& +12\left(m^{2}-9 m+10\right) M_{2}
\end{aligned}
$$

where

$$
\begin{aligned}
M_{2} & =\sum_{1}^{\infty}\left[b_{j}\left(b_{j}+1\right)-a_{j}\left(a_{j}+1\right)\right] \\
& =\sum_{1}^{k}\left[\left(\lambda_{j}-j\right)\left(\lambda_{i}-j+1\right)-j(j-1)\right], \\
M_{2} & =\sum_{1}^{\infty}\left[b_{j}\left(b_{j}+1\right)\left(2 b_{j}+1\right)+a_{j}\left(a_{j}+1\right)\left(2 a_{j}+1\right)\right] \\
& =\sum_{1}^{h}\left[\left(\lambda_{j}-j\right)\left(\lambda_{i}-j+1\right)\left(2 \lambda_{j}-2 j+1\right)+j(j-1)(2 j-1)\right], \\
M_{1} & =\sum_{1}^{\infty}\left[b_{j}^{2}\left(b_{j}+1\right)^{2}-a_{j}^{2}\left(a_{j}+1\right)^{2}\right] \\
& =\sum_{1}^{k}\left[\left(\lambda_{j}-j\right)^{2}\left(\lambda_{j}-j+1\right)^{2}-j^{2}(j-1)^{2}\right],
\end{aligned}
$$




$$
\begin{aligned}
M_{6} & =\sum_{1}^{\infty}\left[b_{j}^{2}\left(b_{j}+1\right)^{2}\left(2 b_{j}+1\right)+a_{j}^{2}\left(a_{j}+1\right)^{2}\left(2 a_{j}+1\right)\right] \\
& =\sum_{1}^{k}\left[\left(\lambda_{j}-j\right)^{2}\left(\lambda_{i}-j+1\right)^{2}\left(2 \lambda_{j}-2 j+1\right)+j^{2}(j-1)^{2}(2 j-1)\right], \\
D_{\lambda} & =\frac{m ! \Delta(l)}{l_{1} ! l_{2} ! \cdots l_{k} !}=\frac{m ! \Delta(b) \Delta(a)}{\prod_{1} b_{j} ! \prod_{1} a_{j} ! \prod_{i, q=1}^{\dot{1}}\left(b_{i}+a_{q}+1\right)} .
\end{aligned}
$$

\section{SElECTEd BibliogRAPHY}

1. G. Frobenius, Uber die Charaktere der symmetrischen Gruppe, Berliner Berichte (1901).

2. - Über die charakteristischen Einheiten der symmetrischen Gruppe, Berliner Berichte (1903).

3. T. Schur, Über die Darstellung der symmetrischen Gruppe durch lineare homogene Substitutionen, Berliner Berichte (1908).

4. F. D. Murnaghan, On the representations of the symmetric group, Amer. J. Math. vol. 59 (1937).

5. - The characters of the symmetric group, Amer. J. Math. vol. 59 (1937).

6. - The theory of group representations, Johns Hopkins Press.

7. J. A. Wheeler, Viewpoints in nuclear structure, Physics Review vol. 52 (1937).

8. E. Wigner, (i) Symmetry of nuclear hamiltonian; Physics Review vol. 52 (1937).

9. - (ii) On the saturation of exchange forces, Proc. Nat. Acad. Sci. U.S.A. vol. 22 (1936) p. 662.

10. H. Weyl, The classical groups, Princeton University Press, 1938.

11. - Theory of groups and quantum mechanics, Dutton.

12. G. de B. Robinson, On the representations of the symmetric group, Amer. J. Math. vol. 60 (1938) pp. 745-760.

13. - On the representations of the symmetric group, Amer. J. Math. vol. 60 (1938).

14. T. Nakayama, On some modular properties of irreducible representations of a symmetric group, Part I, Jap. J. Math. vol. 17 (1940); Part II, ibid.

15. D. E. Littlewood, The theory of group characters, Oxford, 1940.

UNIVERSITY OF IRELAND 